\title{
Physical therapy modalities in neurological disorders at developmental age - Assessment of the methodological value of research papers
}

\author{
Jolanta Zwolińska ${ }^{\mathrm{a}, \mathrm{b}, \mathrm{c}, *}$ and Monika Gąsior ${ }^{\mathrm{a}}$ \\ a St Queen Jadwiga's Regional Clinical Hospital No. 2 in Rzeszow, Rzeszów, Poland \\ ${ }^{\mathrm{b}}$ University of Rzeszow, Institute of Physiotherapy, Faculty of Medicine, Rzeszów, Poland \\ ${ }^{\mathrm{c} C e n t r e ~ f o r ~ I n n o v a t i v e ~ R e s e a r c h ~ i n ~ M e d i c a l ~ a n d ~ N a t u r a l ~ S c i e n c e s, ~ M e d i c a l ~ F a c u l t y ~ o f ~ U n i v e r s i t y ~ o f ~ R z e s z o w, ~}$ \\ Rzeszów, Poland
}

\begin{abstract}
.
BACKGROUND: Physical therapy modalities are often applied in treatment of neurological conditions in children and adolescents.

OBJECTIVE: Evaluation of the methodological quality of research focusing on the application of physical therapy modalities in children and adolescents with neurological conditions.

METHODS: Papers published between 2007 and 2018 were included in the review. 149 papers were analyzed and finally 26 studies investigating the use of physical therapy modalities in children and adolescents with neurological conditions were included in the review. Jadad scale (0-5) was used to assess the methodological value of the studies.

RESULTS: The mean Jadad score was 1.46 (researcher 1) and 1.38 (researcher 2). A score of 0 was awarded to nine (r1) and eight papers (r2). A score of 5 points was awarded to three (r1) and two papers (r2).

CONCLUSION: 1 . The evidence showing the effectiveness of the use of physical therapy modalities is mainly of low quality. 2. The Jadad scale is a valuable tool to assess the quality of research, although it does not always reflect the real value in the case children participate in studies. 3. The analyzed studies show that physical therapy modalities are effective in the treatment of children and adolescents with neurological disorders.
\end{abstract}

Keywords: Neurological diseases, physical therapy modalities, developmental age, Jadad scale, physiotherapy

\section{Introduction}

Due to developments in medicine, continuously increasing numbers of children with neurological disorders are identified. Early implementation of

\footnotetext{
*Address for correspondence: Jolanta Zwolińska, St Queen Jadwiga's Regional Clinical Hospital No. 2 in Rzeszow, ul. Lwowska 60, 35-301 Rzeszów, Poland. Tel.: +48 505095 199; E-mail: jolantazwolinska@op.pl.
}

comprehensive therapy and rehabilitation is essential because of the neuroplasticity of the central nervous system (Panina et al., 2012). Development of optimal therapy is a challenge for the entire medical team (Andruszczak et al., 2012; Garcin, 2018). Physical therapy is often used as an element of modern therapy. Competitiveness of physical therapy is associated with its low invasiveness and limited risk of adverse events (Olchowik et al., 2009; 
Asagai, 2016; Phillips et al., 2016; Olchowik et al., 2010; Zwolińska, 2011; Kwiecień-Czerwieniec \& Woldańska-Okońska, 2012).

Research into the use of physical therapy modalities allows to develop reliable recommendations for the use of physical therapy in clinical practice (Spodaryk \& Bromboszcz, 2004). The methodological quality of a study allows to identify the risk of a biased assessment of treatment effects made by the authors of the study, which is an indispensable step in the process of choosing the best therapy. One of the scales most commonly used to assess the methodological value of research is Jadad scale. This scale has also been adapted for use in many health care areas, including physical therapy (Olivo et al., 200811). It is an easy-to-use tool that is not time-consuming, recommended by other researchers and research institutions (Halpern \& Douglas, 2005; Kaczyński \& Solnica, 2012).

Systematic reviews can identify significant gaps in knowledge and indicate the need for new research (Mancini et al., 2014). Systematic review focusing on physical therapy modalities used in children and adolescents with neurological diseases may be helpful for clinical practitioners in making therapeutic decisions and for researchers implementing projects related to such issues. Literature analysis carried out by us indicates there is a lack of such reviews, therefore we decided to undertake the above subject.

The aim of the review was to assess the methodological quality of research investigating the use of physical therapy modalities in children and adolescents with neurological disorders.

Research questions:

1. What is the value of scientific evidence related to the effectiveness of physical therapy modalities used in children and adolescents with neurological diseases?

2. Is Jadad scale a useful tool for assessing the methodological value of research involving children and adolescents?

3. What is the effectiveness of physical therapy modalities in the treatment of children and adolescents with neurological disorders?

\section{Materials and methods}

The review included papers published between 2007 and 2018 which discussed the use of physical therapy modalities in treatment of children and adolescents with neurological disorders. One of the authors (MG) searched the databases which included Science Direct, SpringerLink, Wiley Online Library, Academic Search Complete, Health Source, Medline Complete, PubMed, and Polish Medical Bibliography. The search was performed using combinations of the following key words: physical therapy, physical therapy modalities, laser, LLLT, LED, magnetotherapy, magnetostimulation, electrotherapy, TENS, NMES, FES, hydrotherapy, cryotherapy, cold therapy, shock wave therapy, child, adolescent, developmental age, and Jadad scale. The review included papers published in various languages (English, Polish, Russian, Portuguese). The following data was extracted from the selected papers: the author and the year of publication, measurements, participants (number, age, sex), details of the intervention (dose, number of sessions, body area), outcomes (short- and long-term effects), side effects, and limitations. Contact with the authors was made when additional data was required.

In total, 149 studies were analyzed. We conducted the selection of papers, and in the case of a disagreement, a consensus was reached through discussion. Papers on nerve conductivity, involving healthy volunteers, descriptions of planned studies and studies underway were excluded from the analysis. Case descriptions and reviews by other authors were omitted. Finally, 26 original papers were evaluated. The attached flowchart presents the rules for the selection of the studies subjected to the evaluation (Fig. 1).

Jadad scale was used to assess the methodological value of the papers. It is a 6-point scale (0-5). Zero rating means the lowest methodological quality, while a rating of five reflects the highest evaluation of a study (Table 1) (Halpern \& Douglas, 2005; Jadad et al., 1996). The evaluation was carried out independently by two researchers (JZ, MG). Compatibility of the assessments by both researchers was tested using Kappa coefficient.

\section{Results}

Of the total 149 papers, 26 met the criteria and were included in the review. The studies on the use of hydrotherapy (one study), cold therapy (three studies), LLLT (three studies), LED therapy (two studies), electrotherapy (eight studies), magnetotherapy (five 


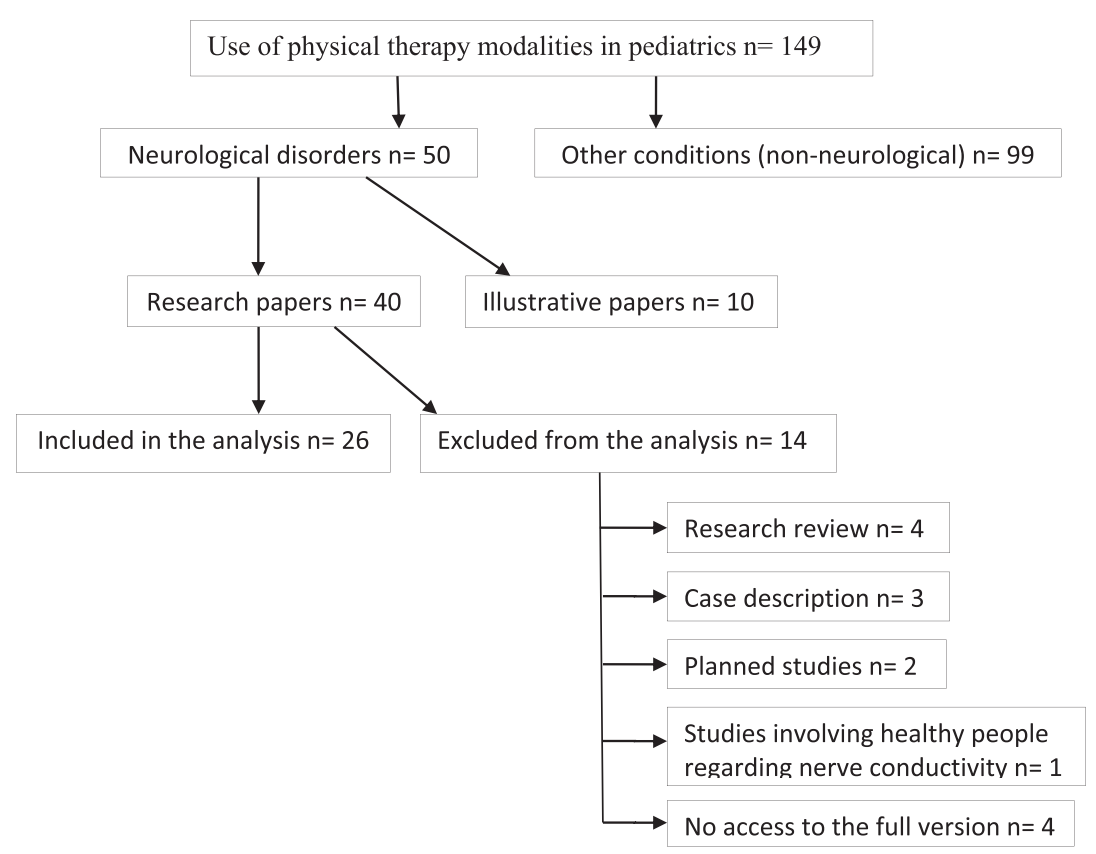

Fig. 1. Flowchart of the procedure.

Table 1

Guidelines for Jadad scale assessment (Jadad et al., 1996; Chung et al., 2012)

\begin{tabular}{lll}
\hline Assessed items & Points & Description \\
\hline Randomization (max.2 points) & $\begin{array}{l}1 \text { point } \\
1 \text { additional point }\end{array}$ & $\begin{array}{l}\text { Randomization mentioned. } \\
\text { Appropriate randomization utilized; } \\
\text { Each study participant has the same chance of receiving each } \\
\text { intervention and the investigators could not predict which } \\
\text { treatment was next: computer-generated random number list, } \\
\text { coin toss, well-shuffled envelopes. }\end{array}$ \\
& 1 point deducted & $\begin{array}{c}\text { Randomization inappropriate: date of birth, date of admission, } \\
\text { hospital number, day of the week. }\end{array}$ \\
Blinding (max.2 points) & $\begin{array}{l}\text { Blinding mentioned. } \\
\text { Appropriate blinding utilized; }\end{array}$ \\
& 1 additional point & $\begin{array}{l}\text { Uses word "double blind", uses active placebos, identical } \\
\text { placebos, or dummies are mentioned. }\end{array}$ \\
Withdrawals and dropouts (max.1 point) & 1 point deducted & $\begin{array}{l}\text { Blinding inappropriate: without placebo or incomplete masking. } \\
\text { Information about participants who have not completed the } \\
\text { study providing reasons or information about no withdrawals. }\end{array}$ \\
\hline
\end{tabular}

studies), and shock wave therapy (four studies) were analyzed. A total of 1069 participants took part in the studies; one paper lacked information on the number of participants included in the study.

Disease entities occurring in the subjects taken into account in the study included cerebral palsy (18 studies), headaches (one study), spina bifida (one study), meningomyelocele (two studies), facial nerve paralysis (one study), ADHD (one study), diabetic polyneuropathy (one study), and psychoautonomic disorder (one study).
Table 2 shows the studies according to the type of physical therapy modality used. Within each physical therapy modality, a chronological order was used.

The results of the papers included in the review indicate that the use of physical therapy modalities in children and adolescents with neurological disorders produces positive results. The review shows that the published papers do not always contain full information about the study participants, the parameters of the procedures performed or the observed side effects of the therapy (Fig. 2). 
Table 2

Analysis of the studies included

\begin{tabular}{|c|c|c|c|c|c|c|c|c|}
\hline Authors & Participants & Treatment & Body area & Outcomes & $\begin{array}{l}\text { Effects } \\
\text { short/long }\end{array}$ & $\begin{array}{l}\text { Combined } \\
\text { therapy }\end{array}$ & $\begin{array}{l}\text { Side } \\
\text { effects }\end{array}$ & Limitation \\
\hline $\begin{array}{l}\text { Hawrylak } \\
\text { (2012) }\end{array}$ & $\begin{array}{l}\text { Cerebral palsy } \\
7 \text { children } \\
15-16 \text { yrs. }\end{array}$ & $\begin{array}{l}\text { Hydromassage } \\
38^{\circ} \mathrm{C}, 20 \text { min., } 1 \\
\text { treatment }\end{array}$ & Whole body & $\begin{array}{l}\text { Improvement of } \\
\text { joint mobility } \\
\text { Reduction of } \\
\text { spasticity } \\
\text { Improved } \\
\text { well-being }\end{array}$ & Short & No & No side effects & $\begin{array}{l}\text { A small study } \\
\text { group }\end{array}$ \\
\hline $\begin{array}{l}\text { Woszczak et } \\
\text { al. (2007) }\end{array}$ & $\begin{array}{l}\mathrm{F}+\mathrm{M} \\
\text { Cerebral palsy } \\
96 \text { children } \\
3-14 \text { yrs. }\end{array}$ & $\begin{array}{l}\text { Cryostimulation (60) } \\
\text { tmax = } 12 \text { min., } 4 \\
\text { weeks } \\
\text { Without } \\
\text { cryostimulation } \\
\text { (36) }\end{array}$ & Lower limbs & $\begin{array}{l}\text { Reduction of } \\
\text { spasticity } \\
\text { Functional } \\
\text { improvement }\end{array}$ & Short & Yes & No side effects & $\begin{array}{l}\text { Lack of } \\
\text { information }\end{array}$ \\
\hline $\begin{array}{l}\text { El Maksound } \\
\text { et al. (2011) }\end{array}$ & $\begin{array}{l}\mathrm{F}+\mathrm{M} \\
\text { Cerebral palsy } \\
30 \text { children } \\
4-6 \text { yrs. }\end{array}$ & $\begin{array}{l}\text { Coldpack (15), } \\
20 \text { min., } 3 \times \text { a week } \\
\text { for } 3 \text { months } \\
\text { Without coldpack (15) }\end{array}$ & $\begin{array}{l}\text { Spastic muscles of } \\
\text { the upper limb }\end{array}$ & $\begin{array}{l}\text { Reduction of } \\
\text { spasticity } \\
\text { Improvement of } \\
\text { the upper limb } \\
\text { function }\end{array}$ & Short & Yes & $\begin{array}{l}\text { Lack of } \\
\quad \text { information }\end{array}$ & $\begin{array}{l}\text { Lack of } \\
\text { information }\end{array}$ \\
\hline $\begin{array}{l}\text { Durairaj et al. } \\
\text { (2018) }\end{array}$ & $\begin{array}{l}\mathrm{F}+\mathrm{M} \\
\text { Cerebral palsy } \\
30 \text { children } \\
3-11 \text { yrs. }\end{array}$ & $\begin{array}{l}\text { Cryotherapy with ice } \\
\text { cubes } \\
20 \text { min., } 3 \times \text { a week } \\
\text { for } 2 \text { months }\end{array}$ & $\begin{array}{c}\text { Muscles of the } \\
\text { upper limbs }\end{array}$ & $\begin{array}{l}\text { Improvement of } \\
\text { the function } \\
\text { Reduction of } \\
\text { spasticity }\end{array}$ & Short & Yes & No side effects & $\begin{array}{l}\text { A single study } \\
\text { group } \\
\text { A small group }\end{array}$ \\
\hline $\begin{array}{l}\text { Asagai et al. } \\
\text { (2007) }\end{array}$ & $\begin{array}{l}\mathrm{F}+\mathrm{M} \\
\text { Cerebral palsy } \\
12 \text { children } \\
15-18 \text { yrs. }\end{array}$ & $\begin{array}{l}\text { LLLT*- }^{*}-810 \mathrm{~nm} \text {, } \\
\text { I irradiation: } 100 \mathrm{~mW} \text {, } \\
\text { 30J } \\
\text { II irradiation: } \\
180 \mathrm{~mW}, 54 \mathrm{~J}\end{array}$ & Carotid arteries & $\begin{array}{l}\text { Increased carotid } \\
\text { artery } \\
\text { cross-section }\end{array}$ & Short & No & $\begin{array}{l}\text { Lack of } \\
\quad \text { information }\end{array}$ & $\begin{array}{l}\text { Lack of } \\
\text { information }\end{array}$ \\
\hline & $\mathrm{F}+\mathrm{M}$ & $1 \times$ & & & & & & \\
\hline
\end{tabular}




\begin{tabular}{|c|c|c|c|c|c|c|c|c|}
\hline $\begin{array}{l}\text { Gottschling et } \\
\text { al. (2008) }\end{array}$ & $\begin{array}{l}\text { Migraine } \\
\quad(22) / \text { Tension- } \\
\text { type } \\
\text { headache } \\
(21) \\
43 \text { children } \\
<17 \text { yrs. } \\
\text { F + M }\end{array}$ & $\begin{array}{l}\text { LLLT } 830 \mathrm{~nm} \text {, } \\
30 \mathrm{~mW}, 3.8 \mathrm{~W} / \mathrm{cm}^{2} \text {, } \\
0.9 \mathrm{~J} / \mathrm{pt} \\
\text { continuous wave } \\
4 \times \text { for } 4 \text { weeks }\end{array}$ & $\begin{array}{l}\text { Acupuncture } \\
\text { points }\end{array}$ & $\begin{array}{l}\text { Reduction in the } \\
\text { frequency of } \\
\text { attacks and the } \\
\text { severity of pain }\end{array}$ & Short & No & $\begin{array}{l}\text { Lack of } \\
\text { information }\end{array}$ & $\begin{array}{l}\text { A small group of } \\
\text { respondents } \\
\text { Lack of evaluation } \\
\text { of long-term } \\
\text { effects }\end{array}$ \\
\hline Asagai (2014) & $\begin{array}{l}\text { Cerebral palsy } \\
4 \text { children } \\
11-17 \text { yrs. }\end{array}$ & $\begin{array}{l}\text { LLLT } 810 \mathrm{~nm} \text {, } \\
9.61 \mathrm{~W} / \mathrm{cm} 2,60 \mathrm{sec} \\
\text { On average } 22 \text { days }\end{array}$ & Spastic muscles & $\begin{array}{l}\text { Improvement of } \\
\text { bone tissue } \\
\text { metabolism (no } \\
\text { density } \\
\text { changes) } \\
\text { Balance of hyper- } \\
\text { parathyroidism }\end{array}$ & Short \& long & No & $\begin{array}{l}\text { Lack of } \\
\text { information }\end{array}$ & $\begin{array}{l}\text { Imperfect bone } \\
\text { density } \\
\text { assessment } \\
\text { methods } \\
\text { A small study } \\
\text { group }\end{array}$ \\
\hline $\begin{array}{l}\text { Asagai et al. } \\
\text { (2012) }\end{array}$ & $\begin{array}{l}\mathrm{F}+\mathrm{M} \\
\text { Cerebral palsy } \\
18 \text { children } \\
2-27 \text { yrs. }\end{array}$ & $\begin{array}{l}\text { LED }^{* *}(8): \\
400-700 \mathrm{~nm}, \\
9.2 \mathrm{~W}, 0.9 \mathrm{~mW} / \mathrm{m}^{2}, \\
825 \mathrm{~lm}, 60 \mathrm{~min} \\
1 \times \text { a day per } 1 \mathrm{month} \\
\text { Without LED }(10)\end{array}$ & Lower limbs & $\begin{array}{l}\text { Improved bone } \\
\text { metabolism }\end{array}$ & Short \& long & No & No side effects & $\begin{array}{l}\text { Lack of } \\
\quad \text { information }\end{array}$ \\
\hline $\begin{array}{l}\text { Asagai et al. } \\
\text { (2014) }\end{array}$ & $\begin{array}{l}\text { Cerebral palsy } \\
82 \text { children } \\
5-18 \text { yrs. } \\
F+M\end{array}$ & $\begin{array}{l}\text { LED }(25): \\
400-700 \mathrm{~nm}, \\
9.2 \mathrm{~W}, 0.9 \mathrm{~mW} / \mathrm{m}^{2}, \\
825 \mathrm{~lm}, 60 \mathrm{~min} \\
1 \times \text { a day per } 1-24 \\
\text { month } \\
\text { Without LED }(57)\end{array}$ & $\begin{array}{l}\text { Upper and lower } \\
\text { limbs }\end{array}$ & $\begin{array}{l}\text { Increased in bone } \\
\text { density }\end{array}$ & Short \& long & No & No side effects & $\begin{array}{l}\text { Discrepancies } \\
\text { between the } \\
\text { irradiation } \\
\text { conditions }\end{array}$ \\
\hline
\end{tabular}


Table 2

(Continued)

\begin{tabular}{|c|c|c|c|c|c|c|c|c|}
\hline Authors & Participants & Treatment & Body area & Outcomes & $\begin{array}{l}\text { Effects } \\
\text { short/long }\end{array}$ & $\begin{array}{l}\text { Combined } \\
\text { therapy }\end{array}$ & $\begin{array}{l}\text { Side } \\
\text { effects }\end{array}$ & Limitation \\
\hline $\begin{array}{l}\text { Cirović et al. } \\
(2009)\end{array}$ & $\begin{array}{l}\text { Spina bifida } \\
\text { and } \\
\text { neurogenic } \\
\text { bladder } \\
49 \text { children } \\
6-12 \text { yrs. } \\
\text { F+ M }\end{array}$ & $\begin{array}{l}\text { TENS } * * *+\text { stimulation } \\
\text { with an exponential } \\
\text { impulse ( } 30): 1 \mathrm{~h} . \\
30 \times \text { per } 1 \text { month } \\
\text { Without } \\
\text { electrotherapy (19) }\end{array}$ & $\begin{array}{l}\text { S2-S4 (TENS) } \\
\text { Suprapubic on the } \\
\text { ventral side } \\
\text { (stimulation) }\end{array}$ & $\begin{array}{l}\text { Improvement of } \\
\text { urodynamic } \\
\text { parameters and } \\
\text { bladder function }\end{array}$ & Long & No & $\begin{array}{l}\text { Lack of } \\
\text { information }\end{array}$ & $\begin{array}{l}\text { Lack of } \\
\quad \text { information }\end{array}$ \\
\hline $\begin{array}{l}\text { Kajbafzadeh } \\
\text { et al. (2011) }\end{array}$ & $\begin{array}{l}\text { Myelomeningocele } \\
\text { with } \\
\text { neurogenic } \\
\text { bowel } \\
\text { dysfunction } \\
30 \text { children } \\
\text { 3-12 yrs. } \\
\text { F+M }\end{array}$ & $\begin{array}{l}\text { Interference currents } \\
(15): \\
\mathrm{fn}=4 \mathrm{kHz} \\
\mathrm{f}_{\text {basic }}=5-25 \mathrm{~Hz} \\
\mathrm{I}_{\max }=50 \mathrm{~mA} \\
20 \mathrm{~min}, 3 \times \text { a week } \\
\text { without } \\
\text { electrotherapy }(15)\end{array}$ & $\begin{array}{l}\text { E: abdomen and } \\
\text { Th12-L4 }\end{array}$ & $\begin{array}{l}\text { Improvement of } \\
\text { the excretory } \\
\text { system function }\end{array}$ & Long & No & No side effects & $\begin{array}{l}\text { Small sample size } \\
\text { and a short } \\
\text { duration of } \\
\text { follow-up }\end{array}$ \\
\hline $\begin{array}{l}\text { Barbaeva et al. } \\
\text { (2014) }\end{array}$ & $\begin{array}{l}\text { Cerebral palsy } \\
168 \text { children } \\
4-15 \text { yrs. }\end{array}$ & $\begin{array}{l}\text { Electrostimulation } \\
\qquad(38): f<1000 \mathrm{~Hz} \\
\text { FES }^{\wedge}(42) \text { : bicycle } \\
\text { training } \\
\text { FES }(45) \text { : during gait } \\
\text { Electrostimulation } \\
(43): f>1000 \mathrm{~Hz} \\
10 \times, 1 \times \text { a day }\end{array}$ & $\begin{array}{l}\text { Dorsal flexors of } \\
\text { the feet }\end{array}$ & $\begin{array}{l}\text { Improvement of } \\
\text { clinical } \\
\text { condition in all } \\
\text { subjects } \\
\text { The best effects } \\
\text { after FES }\end{array}$ & Short & Yes & $\begin{array}{l}\text { Lack of } \\
\text { information }\end{array}$ & $\begin{array}{l}\text { Lack of } \\
\quad \text { information }\end{array}$ \\
\hline $\begin{array}{l}\text { Kajbafzadeh } \\
\text { et al. (2014) }\end{array}$ & $\begin{array}{l}\text { Myelomeningocele } \\
\text { with } \\
\text { incontinence } \\
\text { 30 chilydren } \\
\mathrm{F}+\mathrm{M}\end{array}$ & $\begin{array}{l}\text { FES (15): } \\
t_{\text {imp }}=0.25 \mathrm{~ms} \text {, } \\
15 \mathrm{~Hz}, 15 \mathrm{~min} . \\
\text { without FES (15) }\end{array}$ & $\begin{array}{l}\text { Muscles of the } \\
\text { pelvic floor } \\
\text { Anode: over pubic } \\
\text { symphysis } \\
\text { Cathode: under } \\
\text { the urethra }\end{array}$ & $\begin{array}{l}\text { Improvement of } \\
\text { the urinary } \\
\text { system function }\end{array}$ & Long & No & No side effects & $\begin{array}{l}\text { Lack of } \\
\quad \text { information }\end{array}$ \\
\hline
\end{tabular}




\begin{tabular}{|c|c|c|c|c|c|c|c|c|}
\hline $\begin{array}{l}\text { Arora et al. } \\
\text { (2014) }\end{array}$ & $\begin{array}{l}\text { Cerebral palsy } \\
30 \text { children } \\
4-12 \text { yrs. } \\
\text { F+M }\end{array}$ & $\begin{array}{l}\text { NMES } \$(15) \\
\mathrm{t}_{\mathrm{imp}}=350 \mu \mathrm{s}, \\
\quad \text { rectangular } \\
\text { impulse, } f=30 \mathrm{~Hz}, \\
\text { duty cycle }=50 \% \\
(3 \mathrm{sec} \text { on, } 3 \mathrm{sec} \text { off }) \\
30 \text { min., } 3 \times \text { a week } \\
\text { for } 6 \text { weeks } \\
\text { Without NMES (15) }\end{array}$ & Quadriceps & $\begin{array}{l}\text { Improvement of } \\
\text { the knee } \\
\text { function }\end{array}$ & Short & Yes & $\begin{array}{l}\text { Lack of } \\
\text { information }\end{array}$ & $\begin{array}{l}\text { Lack of } \\
\text { information }\end{array}$ \\
\hline Yang (2015) & $\begin{array}{l}\text { Facial nerve } \\
\quad \text { palsy } \\
60 \text { children } \\
0-14 \text { yrs. } \\
F+M\end{array}$ & $\begin{array}{l}\text { A. Electrostimulation } \\
(50 \mathrm{~Hz})+\mathrm{B} \text {. } \\
\text { Traditional } \\
\text { electrostimulation } \\
(30) \\
6 \text { series of } 10 \\
\text { treatments., } 2 \times \text { a } \\
\text { day } \\
\text { Without } \\
\text { electrotherapy (30) }\end{array}$ & $\begin{array}{l}\text { A. Acupuncture } \\
\text { points }+ \text { B. } \\
\text { Motor points of } \\
\text { facial muscles }\end{array}$ & $\begin{array}{l}\text { Improvement of } \\
\text { facial muscle } \\
\text { function }\end{array}$ & Short & Yes & $\begin{array}{l}\text { Lack of } \\
\text { information }\end{array}$ & $\begin{array}{l}\text { Lack of } \\
\text { information }\end{array}$ \\
\hline $\begin{array}{l}\text { McGough et } \\
\text { al. (2015) }\end{array}$ & $\begin{array}{l}\text { Children with } \\
\text { ADHD } \\
7-14 \text { yrs. } \\
\text { F+M }\end{array}$ & $\begin{array}{l}\text { Electrostimulation } \\
\text { during sleep } \\
\mathrm{t}_{\mathrm{imp}}=250 \mu \mathrm{s}, 120 \mathrm{~Hz} \text {, } \\
\text { duty cycle }=50 \% \\
8 \text { weeks }\end{array}$ & Trigeminal nerve & $\begin{array}{l}\text { Reduction of } \\
\text { ADHD } \\
\text { symptoms and } \\
\text { depression }\end{array}$ & Short & No & $\begin{array}{l}2 \text { children- } \\
\text { headache } \\
1 \text { child- eye } \\
\text { twitching }\end{array}$ & $\begin{array}{l}\text { No control group } \\
\text { and blinding }\end{array}$ \\
\hline $\begin{array}{l}\text { Solopova et al. } \\
\text { (2017) }\end{array}$ & $\begin{array}{l}\text { Cerebral palsy } \\
28 \text { children }\end{array}$ & $\begin{array}{l}\text { Transcutaneous } \\
\text { electrical spinal } \\
\text { cord stimulation } \\
(\mathrm{SCS})(13) \\
\mathrm{t}_{\mathrm{imp}}=1 \mathrm{~ms}, \text { biphasic } \\
\text { rectangular pulses, } \\
\mathrm{f}_{\text {basic }}=30 \mathrm{~Hz}, \\
\mathrm{f}_{\text {supporting }}=10 \mathrm{kHz}\end{array}$ & $\begin{array}{l}\text { Cathodes: midline } \\
\text { Th11-L1 } \\
\text { Anodes: } \\
\text { symmetrically } \\
\text { on the skin over } \\
\text { the iliac crests }\end{array}$ & $\begin{array}{l}\text { Improvement of } \\
\text { motor skills } \\
\text { Reduction } \\
\text { pathological } \\
\text { muscle } \\
\text { co-activation }\end{array}$ & Short & Yes & $\begin{array}{l}\text { Lack of } \\
\text { information }\end{array}$ & $\begin{array}{l}\text { No double } \\
\text { blinding, use of } \\
\text { subjective } \\
\text { assessment } \\
\text { tools, no } \\
\text { repeated } \\
\text { baseline } \\
\text { measures } \\
\end{array}$ \\
\hline
\end{tabular}


Table 2

(Continued)

\begin{tabular}{|c|c|c|c|c|c|c|c|c|}
\hline Authors & Participants & Treatment & Body area & Outcomes & $\begin{array}{l}\text { Effects } \\
\text { short/long }\end{array}$ & $\begin{array}{l}\text { Combined } \\
\text { therapy }\end{array}$ & $\begin{array}{l}\text { Side } \\
\text { effects }\end{array}$ & Limitation \\
\hline & $\begin{array}{l}\text { Mead age } 9.4 \\
\quad \text { yrs. } \\
F+M\end{array}$ & $\begin{array}{l}\mathrm{I}=10-50 \mathrm{~mA} \\
20 \mathrm{~min}, 15 \times \text { per } 3 \\
\quad \text { weeks } \\
\text { Without SCS }(15)\end{array}$ & & $\begin{array}{l}\text { Increase range of } \\
\text { motion }\end{array}$ & & & & \\
\hline \multirow[t]{2}{*}{$\begin{array}{c}\text { Gurova \& } \\
\text { Babina } \\
\text { (2007) }\end{array}$} & $\begin{array}{l}\text { Cerebral palsy } \\
40 \text { children }\end{array}$ & $\begin{array}{l}\text { Magnetoteraphy (20): } \\
33 \mathrm{mT}, 10 \mathrm{~Hz} \\
5-10 \mathrm{~min} ., 8-10 \times\end{array}$ & $\begin{array}{l}\text { Occipital region, } \\
\text { lumbar spine }\end{array}$ & $\begin{array}{l}\text { Improvement of } \\
\text { ENG } \\
\text { parameters } \\
\text { Reduction of } \\
\text { spasticity, } \\
\text { improvement of } \\
\text { intracerebral } \\
\text { circulation }\end{array}$ & Short & Yes & $\begin{array}{l}\text { Lack of } \\
\quad \text { information }\end{array}$ & $\begin{array}{l}\text { Lack of } \\
\text { information }\end{array}$ \\
\hline & $1-7$ yrs & $\begin{array}{l}\text { Without } \\
\text { magnetoteraphy } \\
\text { (20) }\end{array}$ & & & & & & \\
\hline \multirow[t]{2}{*}{$\begin{array}{c}\text { Gurova \& } \\
\text { Babina } \\
(2008)\end{array}$} & Cerebral palsy & $\begin{array}{l}\text { Magnetotherapy (20), } \\
\text { magnetophoresis } \\
(20)\end{array}$ & $\begin{array}{l}\text { Magnetotherapy } \\
\text { for the } \\
\text { sub-occipital } \\
\text { area and lumbar } \\
\text { section Magne- } \\
\text { tophoresis ( } 2 \% \\
\text { glutamic acid: } \\
\text { sub-occipital } \\
\text { area,2\% } \\
\text { magnesium } \\
\text { sulfate: L-S }\end{array}$ & $\begin{array}{l}\text { Improvement of } \\
\text { sleep, reduction } \\
\text { of spasticity and } \\
\text { clonus }\end{array}$ & Short & Yes & $\begin{array}{l}\text { Lack of } \\
\text { information }\end{array}$ & $\begin{array}{l}\text { Lack of } \\
\quad \text { information }\end{array}$ \\
\hline & 40 children & $\begin{array}{l}33 \mathrm{mT}, 10 \mathrm{~Hz}, \\
5-10 \mathrm{~min} ., 14 \text { days }\end{array}$ & & $\begin{array}{l}\text { Better effects after } \\
\text { magnetophore- } \\
\text { sis }\end{array}$ & & & & \\
\hline \multirow[t]{3}{*}{$\begin{array}{l}\text { Nikolaeva et } \\
\text { al. (2010) }\end{array}$} & $\begin{array}{l}\text { 1-7 yrs. } \\
\text { Diabetic } \\
\text { polyneu- } \\
\text { ropathy }\end{array}$ & Magnetotherapy (23): & Lower limbs & $\begin{array}{r}\text { Paresthesia } \\
\text { reduction }\end{array}$ & Short & No & $\begin{array}{l}\text { Lack of } \\
\text { information }\end{array}$ & $\begin{array}{l}\text { Lack of } \\
\text { information }\end{array}$ \\
\hline & 45 children & $\begin{array}{l}45 \mathrm{mT} \text {, sinusoidal } \\
\text { impulse, } \\
15 \text { min., } 10 \times\end{array}$ & & $\begin{array}{l}\text { Improved } \\
\text { microcirculation } \\
\text { and oxygenation } \\
\text { of the tissue }\end{array}$ & & & & \\
\hline & $5-17$ yrs. & & & $\begin{array}{l}\text { Improvement of } \\
\text { ENG } \\
\text { parameters in } \\
\text { motor fibers }\end{array}$ & & & & \\
\hline
\end{tabular}


Table 2

(continued)

\begin{tabular}{|c|c|c|c|c|c|c|c|c|}
\hline Authors & Participants & Treatment & Body area & Outcomes & $\begin{array}{l}\text { Effects } \\
\text { short/long }\end{array}$ & $\begin{array}{l}\text { Combined } \\
\text { therapy }\end{array}$ & $\begin{array}{l}\text { Side } \\
\text { effects }\end{array}$ & Limitation \\
\hline & $\mathrm{F}+\mathrm{M}$ & $\begin{array}{l}\text { Without } \\
\text { magnetoteraphy } \\
(22)\end{array}$ & & & & & & \\
\hline \multirow[t]{4}{*}{$\begin{array}{l}\text { Filina et al. } \\
\quad(2010)\end{array}$} & $\begin{array}{l}\text { Type I } \\
\text { diabetes } \\
\text { with } \\
\text { symptoms } \\
\text { of } \\
\text { aggression, } \\
\text { depression } \\
\text { and anxiety }\end{array}$ & $\begin{array}{l}\text { Transcranial } \\
\text { magnetotherapy } \\
(40)\end{array}$ & Head (headband) & $\begin{array}{l}\text { Improvement of } \\
\text { the psychoemo- } \\
\text { tional state and } \\
\text { functions of the } \\
\text { autonomic } \\
\text { system }\end{array}$ & Short & No & $\begin{array}{l}\text { Lack of } \\
\quad \text { information }\end{array}$ & $\begin{array}{l}\text { Lack of } \\
\quad \text { information }\end{array}$ \\
\hline & 80 children & $\begin{array}{c}10-30 \mathrm{mT}, 1-10 \mathrm{~Hz}, \\
7-12 \mathrm{~min} .10 \times\end{array}$ & & & & & & \\
\hline & $7-17$ yrs. & & & & & & & \\
\hline & & $\begin{array}{l}\text { Without } \\
\text { magnetoteraphy } \\
(40)\end{array}$ & & & & & & \\
\hline \multirow[t]{4}{*}{$\begin{array}{l}\text { Panina et al. } \\
\text { (2012) }\end{array}$} & $\begin{array}{l}\text { Perinatal } \\
\text { damage to } \\
\text { the CNS }\end{array}$ & $\begin{array}{l}\text { Transcranial } \\
\text { magnetotherapy } \\
(40)\end{array}$ & $\begin{array}{l}\text { Head (neonate } \\
\text { headband) }\end{array}$ & $\begin{array}{l}\text { Improvement of } \\
\text { blood flow } \\
\text { within the brain }\end{array}$ & Short & Yes & $\begin{array}{l}\text { Lack of } \\
\quad \text { information }\end{array}$ & $\begin{array}{l}\text { Lack of } \\
\quad \text { information }\end{array}$ \\
\hline & 80 children & $\begin{array}{l}15 \mathrm{mT}, 10 \mathrm{~Hz}, \\
5-10 \mathrm{~min} ., 10 \times\end{array}$ & & $\begin{array}{l}\text { Reduction of } \\
\text { hyperactivity } \\
\text { and disorders of } \\
\text { the autonomic } \\
\text { nervous system }\end{array}$ & & & & \\
\hline & $\begin{array}{l}14 \text { days- } 4 \\
\text { months }\end{array}$ & & & & & & & \\
\hline & $\mathrm{F}+\mathrm{M}$ & $\begin{array}{l}\text { Without } \\
\text { magnetoteraphy } \\
(40)\end{array}$ & & & & & & \\
\hline \multirow[t]{3}{*}{$\begin{array}{l}\text { Amelio \& } \\
\quad \text { Manganotti } \\
\text { (2010) }\end{array}$} & Cerebral palsy & Shockwaves & $\begin{array}{l}\text { Plantar foot } \\
\text { flexors }\end{array}$ & $\begin{array}{l}\text { Lowering muscle } \\
\text { tone (up to } \\
\text { several weeks) }\end{array}$ & Short \& long & Yes & No side effects & $\begin{array}{l}\text { Lack of double } \\
\text { blinding }\end{array}$ \\
\hline & 12 children & $\begin{array}{l}1500 \text { hits, } \\
0.03 \mathrm{~mJ} / \mathrm{mm} 2,1 \times\end{array}$ & & & & & & \\
\hline & $\begin{array}{l}6-11 \text { yrs. } \\
F+M\end{array}$ & & & & & & & \\
\hline
\end{tabular}


Table 2

\begin{tabular}{|c|c|c|c|c|c|c|c|c|}
\hline Authors & Participants & Treatment & Body area & Outcomes & $\begin{array}{l}\text { Effects } \\
\text { short/long }\end{array}$ & $\begin{array}{l}\text { Combined } \\
\text { therapy }\end{array}$ & $\begin{array}{l}\text { Side } \\
\text { effects }\end{array}$ & Limitation \\
\hline $\begin{array}{l}\text { Vidala et al. } \\
\text { (2011) }\end{array}$ & $\begin{array}{l}\text { Cerebral palsy } \\
15 \text { individuals } \\
10-46 \text { yrs. } \\
F+M\end{array}$ & $\begin{array}{l}\text { Shockwaves } \\
\begin{array}{l}2 \text { bars, } 2000 \text { hits, } \\
8 \mathrm{~Hz}, 0.10 \mathrm{~mJ} / \mathrm{mm} 2 \text {, } \\
3 \times\end{array}\end{array}$ & $\begin{array}{l}14 \text { spastic muscles } \\
13 \text { antagonists of } \\
\text { spastic muscles }\end{array}$ & $\begin{array}{l}\text { Lowering muscle } \\
\text { tone } \\
\text { Improving the } \\
\text { range of } \\
\text { movement }\end{array}$ & Short \& long & No & $\begin{array}{l}3 \text { children: } \\
\text { hematomas, } \\
\text { effusions, } \\
\text { pain }\end{array}$ & $\begin{array}{l}\text { Lack of } \\
\quad \text { information }\end{array}$ \\
\hline $\begin{array}{l}\text { Gonkova et al. } \\
\text { (2013) }\end{array}$ & $\begin{array}{l}\text { Cerebral palsy } \\
25 \text { children } \\
\text { Mean age } \\
\quad 4.84 \text { yrs. } \\
\text { F+ M }\end{array}$ & Radial shockwave $1 \times$ & $\begin{array}{l}\text { Plantar foot } \\
\text { flexors }\end{array}$ & $\begin{array}{l}\text { Lowering } \\
\text { spasticity } \\
\text { An increase in the } \\
\text { contact surface } \\
\text { area of the foot } \\
\text { with the ground } \\
\text { Higher heel } \\
\text { pressure } \\
\text { (minimum } 4 \\
\text { weeks) }\end{array}$ & Short & No & $\begin{array}{l}\text { Lack of } \\
\quad \text { information }\end{array}$ & $\begin{array}{l}\text { No evaluation of } \\
\text { long-time } \\
\text { effects, the } \\
\text { study and } \\
\text { control groups } \\
\text { were the same } \\
\text { children, a small } \\
\text { number of } \\
\text { respondents }\end{array}$ \\
\hline $\begin{array}{l}\text { Gawad et al. } \\
\text { (2015) }\end{array}$ & $\begin{array}{l}\text { Cerebral palsy } \\
15 \text { children } \\
5-7 \text { yrs. } \\
F+M\end{array}$ & $\begin{array}{l}\text { Shockwave } \\
3 \times \text { a week }\end{array}$ & $\begin{array}{l}\text { Plantar foot } \\
\text { flexors }\end{array}$ & $\begin{array}{l}\text { Decreasing } \\
\text { muscle tone } \\
\text { Improvement of } \\
\text { locomotor } \\
\text { abilities }\end{array}$ & Long & Yes & $\begin{array}{l}\text { Lack of } \\
\quad \text { information }\end{array}$ & $\begin{array}{l}\text { Lack of } \\
\quad \text { information }\end{array}$ \\
\hline
\end{tabular}

Electrical Stimulation. 


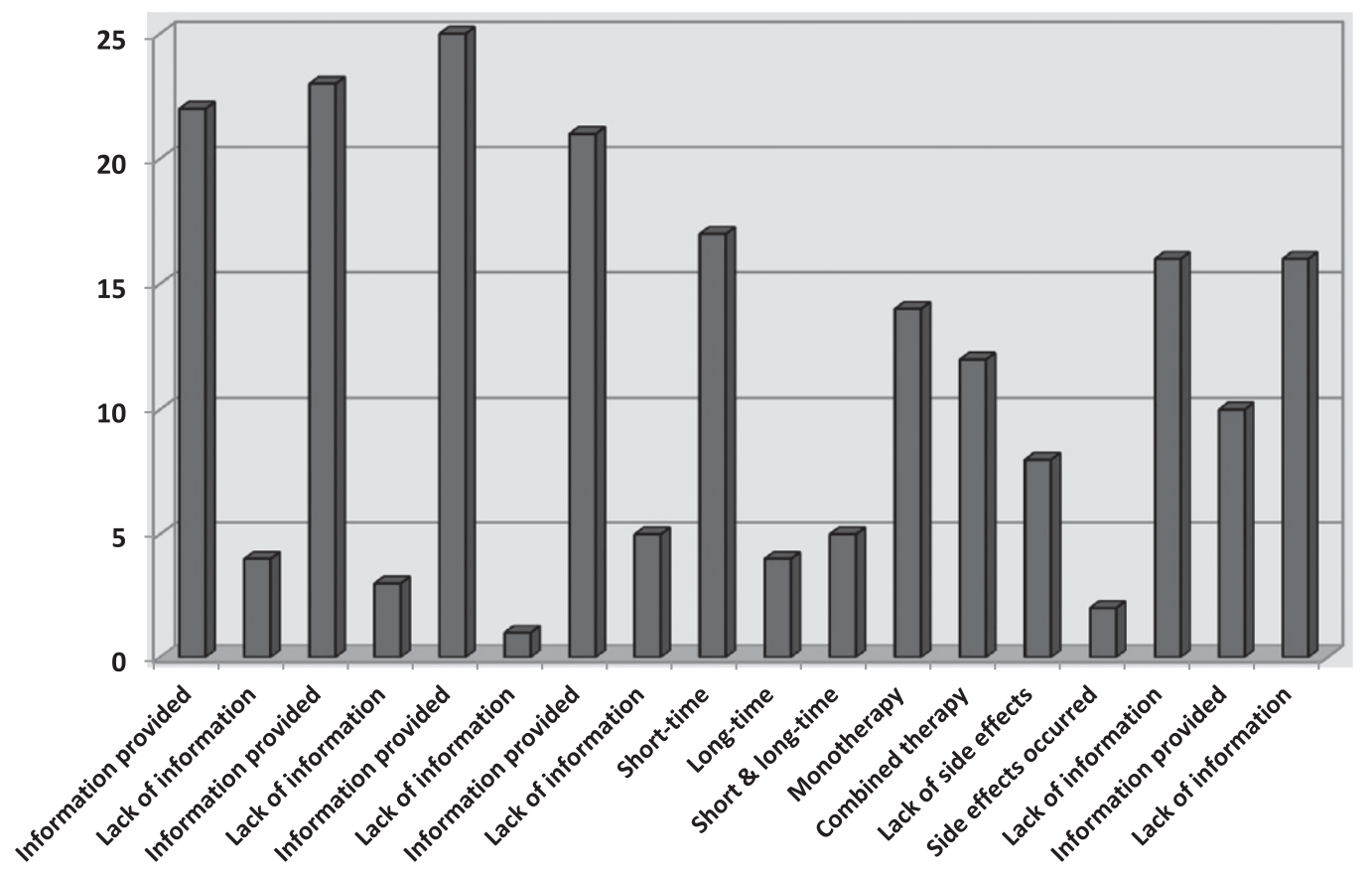

Fig. 2. Analysis of the included papers.

\section{Assessment of the methodological value of the papers with Jadad scale}

Below is the score given by both researchers for the individual papers (Table 3 ) as well as a summary of the ratings by both researchers (Fig. 3).

The calculated Kappa coefficient showed almost complete agreement between the two researchers (JZ, MG) in the overall Jadad score (Kappa coefficient $=0.92$ ). The relationship was statistically significant $(p<0.001)$. A highly statistically significant correlation between the analyzed variables (Spearman's rho $=0.94, p<0.001$ ) was also found (Table 4).

We found full agreement between the researchers in the scoring for randomization (Kappa coefficient $=1, p<0.001)$, as well as correct / incorrect randomization (Kappa coefficient $=1, p<0.001$ ). The researchers' opinions were also fully compatible in the rating of the blinding of the study (Kappa coefficient $=1, p<0.001$ ).

Nearly full agreement between the researchers was also found in their scoring for correct/incorrect blinding $($ Kappa coefficient $=0.91, p<0.001)($ Table 5).

Nearly full agreement between the researchers was shown in the score given for information about participants who dropped out from the study (Kappa coefficient $=0.82, p<0.001)$ (Table 6).
The arithmetic means of Jadad scale ratings by the 1 st researcher (JZ) amounted to 1.46 and by the 2 nd researcher (MG) equalled 1.38. The median for the evaluations by both researchers amounted to one.

\section{Discussion}

Most physical factors can be used to treat disorders and dysfunctions of the nervous system regardless of the patient's age (Zwolińska, 2011; KwiecieńCzerwieniec \& Woldańska-Okońska, 2012). However, there are some deficiencies in the standards of physical therapy in patients at developmental age (Phillips et al., 2016; de Sá \& Gomes, 2013).

\subsection{Evidence-based medicine (EBM)}

The progress in medicine depends on the implementation of high-quality research (Almeida et al., 2018). Research planned and implemented in accordance with the requirements of evidence-based medicine (EBM) allows to document the effectiveness of physiotherapeutic methods, and this in turn is an indispensable condition for development in the field of physical medicine and rehabilitation

(Olchowik et al., 2010; Zwolińska, 2011; Kaczyński \& Solnica, 2012; Mancini et al., 2014). 
Table 3

Scoring in Jadad scale for individual papers

\begin{tabular}{|c|c|c|c|c|c|c|c|c|c|c|c|c|}
\hline \multirow[t]{2}{*}{$\begin{array}{l}\text { Author/ } \\
\text { year }\end{array}$} & \multicolumn{2}{|c|}{ Randomization } & \multicolumn{2}{|c|}{$\begin{array}{c}\text { Correct/ } \\
\text { incorrect } \\
\text { randomization }\end{array}$} & \multicolumn{2}{|c|}{ Blinding } & \multicolumn{2}{|c|}{$\begin{array}{l}\text { Correct/ } \\
\text { incorrect } \\
\text { blinding }\end{array}$} & \multicolumn{2}{|c|}{$\begin{array}{c}\text { Information } \\
\text { about } \\
\text { dropouts }\end{array}$} & \multicolumn{2}{|c|}{$\begin{array}{c}\text { Overall } \\
\text { score } \\
\text { Jadad scale }\end{array}$} \\
\hline & $\mathrm{JZ}$ & MG & $\mathrm{JZ}$ & MG & $\mathrm{JZ}$ & MG & $\mathrm{JZ}$ & MG & $\mathrm{JZ}$ & MG & $\mathrm{JZ}$ & MG \\
\hline Hawrylak (2012) & 0 & 0 & 0 & 0 & 0 & 0 & 0 & 0 & 1 & 1 & $1 / 5$ & $1 / 5$ \\
\hline Woszczak et al. (2007) & 0 & 0 & 0 & 0 & 0 & 0 & 0 & 0 & 0 & 0 & $0 / 5$ & $0 / 5$ \\
\hline El Maksound et al. (2011) & 1 & 1 & 0 & 0 & 1 & 1 & 1 & -1 & 0 & 0 & $3 / 5$ & $1 / 5$ \\
\hline Durairaj et al. (2018) & 0 & 0 & 0 & 0 & 0 & 0 & 0 & 0 & 0 & 0 & $0 / 5$ & $0 / 5$ \\
\hline Asagai et al. (2007) & 0 & 0 & 0 & 0 & 0 & 0 & 0 & 0 & 0 & 1 & $0 / 5$ & $1 / 5$ \\
\hline Gottschling et al. (2008) & 1 & 1 & 1 & 1 & 1 & 1 & 1 & 1 & 1 & 1 & $5 / 5$ & $5 / 5$ \\
\hline Asagai (2014) & 0 & 0 & 0 & 0 & 0 & 0 & 0 & 0 & 0 & 0 & $0 / 5$ & $0 / 5$ \\
\hline Asagai et al. (2012) & 0 & 0 & 0 & 0 & 0 & 0 & 0 & 0 & 1 & 1 & $1 / 5$ & $1 / 5$ \\
\hline Asagai et al. (2014) & 0 & 0 & 0 & 0 & 0 & 0 & 0 & 0 & 0 & 0 & $0 / 5$ & $0 / 5$ \\
\hline Cirović et al. (2009) & 1 & 1 & 0 & 0 & 0 & 0 & 0 & 0 & 0 & 0 & $1 / 5$ & $1 / 5$ \\
\hline Kajbafzadeh et al. (2011) & 1 & 1 & 0 & 0 & 1 & 1 & 0 & 0 & 0 & 0 & $2 / 5$ & $2 / 5$ \\
\hline Barbaeva et al. (2014) & 0 & 0 & 0 & 0 & 0 & 0 & 0 & 0 & 0 & 0 & $0 / 5$ & $0 / 5$ \\
\hline Kajbafzadeh et al. (2014) & 1 & 1 & 1 & 1 & 1 & 1 & 1 & 1 & 1 & 0 & $5 / 5$ & $4 / 5$ \\
\hline Arora et al. (2014) & 1 & 1 & 1 & 1 & 0 & 0 & 0 & 0 & 1 & 1 & $3 / 5$ & $3 / 5$ \\
\hline Yang (2015) & 1 & 1 & 1 & 1 & 0 & 0 & 0 & 0 & 1 & 1 & $3 / 5$ & $3 / 5$ \\
\hline McGough et al. (2015) & 0 & 0 & 0 & 0 & 0 & 0 & 0 & 0 & 1 & 1 & $1 / 5$ & $1 / 5$ \\
\hline Solopova et al. (2017) & 1 & 1 & 0 & 0 & 1 & 1 & -1 & -1 & 0 & 0 & $1 / 5$ & $1 / 5$ \\
\hline Gurova \& Babina (2007) & 0 & 0 & 0 & 0 & 1 & 1 & 0 & 0 & 0 & 0 & $1 / 5$ & $1 / 5$ \\
\hline Gurova \& Babina (2008) & 0 & 0 & 0 & 0 & 0 & 0 & 0 & 0 & 0 & 0 & $0 / 5$ & $0 / 5$ \\
\hline Nikolaeva et al. (2010) & 0 & 0 & 0 & 0 & 1 & 1 & 0 & 0 & 0 & 0 & $1 / 5$ & $1 / 5$ \\
\hline Filina et al. (2010) & 1 & 1 & 0 & 0 & 1 & 1 & 0 & 0 & 0 & 0 & $2 / 5$ & $2 / 5$ \\
\hline Panina et al. (2012) & 0 & 0 & 0 & 0 & 0 & 0 & 0 & 0 & 0 & 0 & $0 / 5$ & $0 / 5$ \\
\hline Amelio \& Manganotti (2010) & 0 & 0 & 0 & 0 & 1 & 1 & -1 & -1 & 0 & 0 & $0 / 5$ & $0 / 5$ \\
\hline Vidala et al. (2011) & 1 & 1 & 1 & 1 & 1 & 1 & 1 & 1 & 1 & 1 & $5 / 5$ & $5 / 5$ \\
\hline Gonkova et al. (2013) & 0 & 0 & 0 & 0 & 1 & 1 & 1 & 1 & 0 & 0 & $2 / 5$ & $2 / 5$ \\
\hline Gawad et al. (2015) & 1 & 1 & 0 & 0 & 0 & 0 & 0 & 0 & 0 & 0 & $1 / 5$ & $1 / 5$ \\
\hline
\end{tabular}

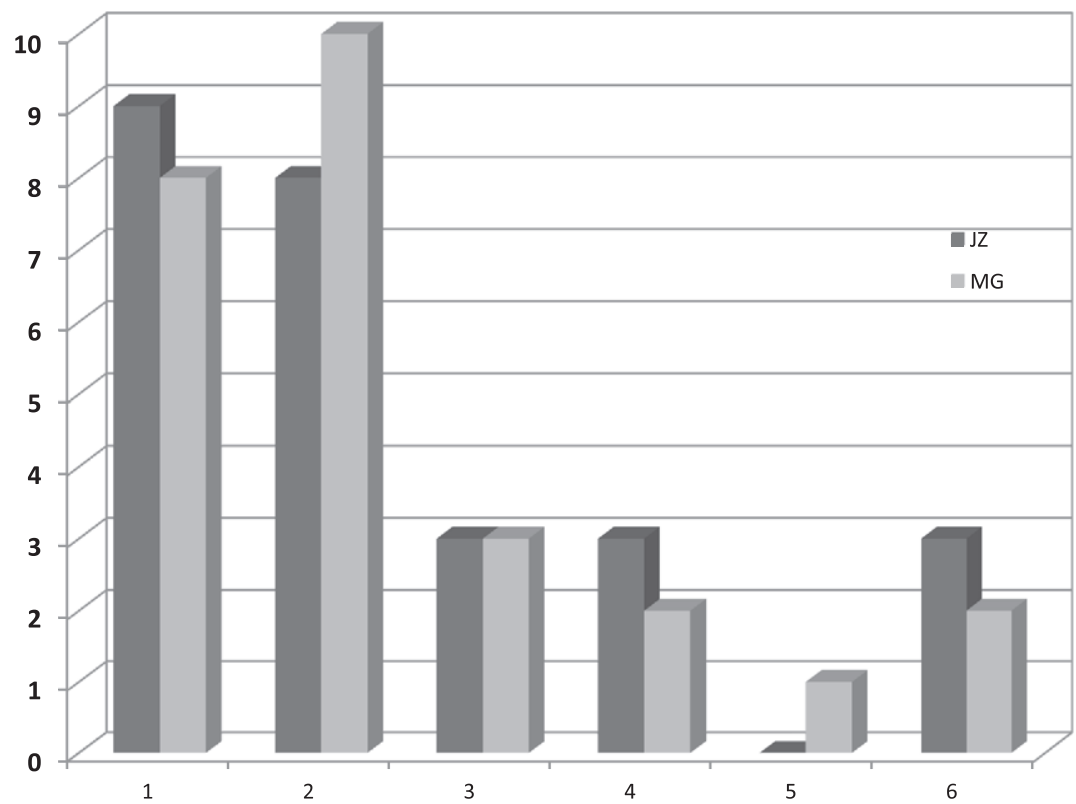

Fig. 3. Comparison of the results of the assessments by both researchers. 
Table 4

The relationship between the general Jadad scores for both researchers

\begin{tabular}{|c|c|c|c|c|c|c|}
\hline \multicolumn{7}{|c|}{$\begin{array}{c}\text { Score in Jadad scale }(0-5) \\
\text { Cross table MG } * \text { JZ }\end{array}$} \\
\hline & & & \multicolumn{3}{|c|}{$\mathrm{JZ}$} & \multirow[t]{2}{*}{ Total } \\
\hline & & & score $0-1$ & score $2-3$ & score $4-5$ & \\
\hline \multirow[t]{8}{*}{ MG } & score $0-1$ & $\mathrm{~N}$ & 17 & 1 & 0 & 18 \\
\hline & & $\%$ & $100.0 \%$ & $16.7 \%$ & $.0 \%$ & $69.2 \%$ \\
\hline & score $2-3$ & $\mathrm{~N}$ & 0 & 5 & 0 & 5 \\
\hline & & $\%$ & $0.00 \%$ & $83.3 \%$ & $0.00 \%$ & $19.2 \%$ \\
\hline & score $4-5$ & $\mathrm{~N}$ & 0 & 0 & 3 & 3 \\
\hline & \multirow{3}{*}{ Total } & $\%$ & $0.00 \%$ & $0.00 \%$ & $100.0 \%$ & $11.5 \%$ \\
\hline & & $\mathrm{N}$ & 17 & 6 & 3 & 26 \\
\hline & & $\%$ & $100.0 \%$ & $100.0 \%$ & $100.0 \%$ & $100.0 \%$ \\
\hline
\end{tabular}

$p<0.001$, Kappa $=0.92 ; p<0.001$, Spearman's rho $=0.94$.

Table 5

Relationship between Jadad scores for correct/incorrect blinding of the studies for both researchers

\begin{tabular}{|c|c|c|c|c|c|c|}
\hline \multicolumn{7}{|c|}{$\begin{array}{l}\text { Correct/incorrect blinding } \\
\text { Cross table } \mathrm{MG} * \mathrm{JZ}\end{array}$} \\
\hline & & & \multicolumn{3}{|c|}{$\mathrm{JZ}$} & \multirow[t]{2}{*}{ Total } \\
\hline & & & score -1 & score 0 & score 1 & \\
\hline \multirow[t]{8}{*}{ MG } & score -1 & $\mathrm{~N}$ & 2 & 0 & 1 & 3 \\
\hline & & $\%$ & $100.0 \%$ & $0.00 \%$ & $20.0 \%$ & $11.5 \%$ \\
\hline & score 0 & $\mathrm{~N}$ & 0 & 19 & 0 & 19 \\
\hline & & $\%$ & $0.00 \%$ & $100.0 \%$ & $0.00 \%$ & $73.1 \%$ \\
\hline & score 1 & $\mathrm{~N}$ & 0 & 0 & 4 & 4 \\
\hline & \multirow{3}{*}{ Total } & $\%$ & $0.00 \%$ & $0.00 \%$ & $80.0 \%$ & $15.4 \%$ \\
\hline & & $\mathrm{N}$ & 2 & 19 & 5 & 26 \\
\hline & & $\%$ & $100.0 \%$ & $100.0 \%$ & $100.0 \%$ & $100.0 \%$ \\
\hline
\end{tabular}

$p<0.001$, Kappa $=0.91$.

Table 6

Relationship between Jadad scores for information about participants who dropped out from the study, for both researchers

\begin{tabular}{|c|c|c|c|c|c|}
\hline \multicolumn{6}{|c|}{$\begin{array}{l}\text { Information about participants who dropped out from } \\
\text { the study } \\
\text { Cross table MG * JZ }\end{array}$} \\
\hline & & & \multicolumn{2}{|c|}{$\mathrm{JZ}$} & \multirow[t]{2}{*}{ Total } \\
\hline & & & score 0 & score 1 & \\
\hline \multirow[t]{6}{*}{ MG } & score 0 & $\mathrm{~N}$ & 17 & 1 & 18 \\
\hline & & $\%$ & $94.4 \%$ & $12.5 \%$ & $69.2 \%$ \\
\hline & score 1 & $\mathrm{~N}$ & 1 & 7 & 8 \\
\hline & & $\%$ & $5.6 \%$ & $87.5 \%$ & $30.8 \%$ \\
\hline & Total & $\mathrm{N}$ & 18 & 8 & 26 \\
\hline & & $\%$ & $100.0 \%$ & $100.0 \%$ & $100.0 \%$ \\
\hline
\end{tabular}

$p<0.001$, Kappa $=0.82$.

Randomized clinical trials (RCTs) are the basic source of scientific evidence confirming or not the effectiveness of various therapies (Kaczyński \& Solnica, 2012; Möller, 2011).

Analysis of systematic reviews often indicates a lack of a sufficient number of randomized trials. However, appropriate assessment of the quality of non-randomized trials can provide valuable scientific data (Kaczyński \& Solnica, 2012).

\subsection{Jadad scale}

Many scales are used to assess the methodological quality of research. The risk of biased assessment is reduced by the use of standardized tools that include Jadad scale (Olivo et al., 2008; Kaczyński \& Solnica, 2012). This scale is most often applied but its usefulness is questioned by some researchers.

In this systematic review, two independent authors assessed the methodological quality of 26 studies, using Jadad scale. All the studies focused on application of physical factors in patients at developmental age with nervous system diseases or dysfunctions. The results of the conducted research indicate good effects of physical therapy, although the methodological quality of the conducted tests is not high.

Chung et al. (2012) interprets a score $\geq 3$ on Jadad scale as indicative of a high-quality study, whereas a result $\leq 2$ reflects a poor-quality study (Chung 
et al., 2012). According to such classification, 20 (JZ) and 21 (MG) studies included in our review present poor methodological quality. Only six (JZ) and five (MG) studies are of high methodological quality.

Simon (2006) suggests that a score of $\leq 3$ on Jadad scale reflects poor reliability of evidence reported by a study regarding the effects of a given intervention (Simon, 2006). In our review, as many as 23 out of the total 26 studies considered, obtained score $\leq 3$, which allows us to conclude that there is very unreliable evidence related to effectiveness of physical factors used in treatment of children and adolescents with neurological disorders.

Chung et al. (2009), Lee et al. (2011) as well as Chung et al. (2012) used Jadad scale to evaluate medical research (Chung et al., 2009, 2012; Lee et al., 2011). Chung et al. (2009) obtained the mean Jadad score of 1.87. In the study by Lee et al. (2011) this result was 1.75 . In the study by Chung et al. (2012), the mean rating of the studies amounted to 1.33 for the years 1991-1995, and 3.00 for 2011. In our review, the mean Jadad score was 1.46 (JZ) and 1.38 (MG).

The low methodological value of the studies included in our review resulted from the lack of randomization, blinding and incomplete information about cases of non-completion of the study. Approximately one in three of the analyzed studies scored 0 in Jadad scale according to both researchers (JZ, MG).

Notably, a correct design of randomized trials is sometimes impossible due to organizational reasons. An ethical aspect of trials involving control groups treated with placebo also needs consideration (Kaczyński \& Solnica, 2012).

Implementation of double blinded studies reduces the risk of unreliability of researchers and allows to avoid research bias (Spodaryk \& Bromboszcz, 2004). Blinding of the researcher and the evaluator is possible and even desirable, while blinding of a small child or a child with difficult contact does not always seem necessary for the examination. Double blinding is not always possible due to the nature of the interventions (Olivo et al., 2008).

The element that most often made it impossible for a study to obtain a maximum Jadad score was the lack of information about the participants who dropped out from the study. If the data on the size of the examined group were included in a table, while the text contained no information about the reasons for the non-completion of the study by individual subjects, the study obtained a lower score. Accord- ing to the authors of this review, if discontinuing the study was not related to the therapy, then, with a sufficiently large group of respondents, other reasons for exclusion are not significant for the quality of the study.

\subsection{Limitation of the assessed papers}

In the case of diseases and disorders frequently occurring in a population, the groups included in the study should be sufficiently large (Spodaryk \& Bromboszcz, 2004). Inclusion of small sample sizes in a study is linked with a greater risk of false results (Spodaryk \& Bromboszcz, 2004; Olivo et al., 2008; Kaczyński \& Solnica, 2012). It is noticeable in this review that very small groups of participants were often included, despite the prevalence of diseases.

The lack of blinding affects the internal reliability of the study (Mancini et al., 2014). Only in five (JZ) and four (MG) studies assessed, correct blinding was performed, which increases the risk of biased assessment in the tests selected for the review. If a physical modality procedure is not easily recognizable, it is possible to perform a blinding.

In some of the studies considered, there is no information about the parameters and methodology of the procedure. In order for publications on physical modalities to stimulate the development of clinical practice and contribute to the improvement of the treatment process, it is necessary to consider information on the parameters of physical therapy performed. Also, according to Galea (2012), such information is extremely valuable (Galea, 2012).

In 16 papers, the authors did not provide information about the occurrence or absence of side effects of therapy. These elements are important for physiotherapists in clinical practice. However, in the case of young children or patients with difficult contact, the effectiveness of the treatment and possible side effects may be difficult to assess.

\subsection{Strengths and limitations of the present review}

The strength of our review lies in the fact that an independent assessment was carried out by two researchers. In the case of any doubts, the reviewers re-verified the texts of the papers. In addition, e-mail contact with authors of papers was initiated in order to clarify doubts that have a significant impact on the methodological value of a study. In order to supplement and update the literature, the search of databases was repeated several times. 
Given the fact that various physical factors were investigated and different methods were applied in assessing effectiveness of the therapies in the relevant studies, no meta-analysis was carried out. The limited number of papers included is a drawback of this review. There is a risk that during a search of literature a publication may be omitted due to the fact that the physical factor modality is not always included in the key words. Also, several different papers by the same author were included regarding light therapy, which may pose a risk of this author's bias.

\section{Conclusion}

1. The evidence related to the effectiveness of the use of physical therapy modalities in treatment of children and adolescents with neurological disorders was predominantly found to be of low quality.

2. Jadad scale is a valuable tool enabling assessment of the quality of research, although it does not always reflect the real value of studies in the case of research involving children and adolescents.

3. The analyzed studies show that physical therapy modalities are effective in the treatment of children and adolescents with neurological disorders.

The authors of this review recognize a need for:

- Continuation of well-designed research, with high methodological quality, investigating the use of physical therapy modalities in children and adolescents with neurological diseases

- Carrying out systematic reviews including a critical evaluation of such tests

\section{Conflict of interest}

None to report.

\section{References}

Almeida, C.C., Silva, V.Z.M.D., Júnior, G.C., Liebano, R.E., \& Durigan, J.L.Q. (2018). Transcutaneous electrical nerve stimulation and interferential current demonstrate similar effects in relieving acute and chronic pain: a systematic review with meta-analysis. Brazilian Journal of Physical Therapy, 22, 347354. doi: 10.1016/j.bjpt.2017.12.005
Amelio, E., \& Manganotti, P. (2010). Effect of shock wave stimulation on hypertonic plantar flexor muscles in patients with cerebral palsy: a placebo-controlled study. Journal of Rehabilitation Medicine, 42, 339-343. doi: 10.2340/16501977-0522

Andruszczak, B., Buraczyńska-Andrzejewska, B., Krauss, H., Jończyk-Potoczna, K., Piątek, J., Krzywicka, A., ŻukiewiczSobczak, W., Krasowska, E., \& Kozak, M. (2012). Multi-level care of a child with cerebral palsy. Medycyna Ogólna i Nauki o Zdrowiu, 18, 314-318.

Arora, L., Arora, R., \& Kataria, V. (2014). Effect of electrical stimulation on spasticity in spastic diplegic cerebral palsy children. International Journal of Innovative Research \& Development, 3, 395-400.

Asagai, Y. (2014). The effect of LLLT on bone metabolism in children with severe cerebral palsy (a secondary publication). Laser Therapy, 23, 243-247. doi: 10.5978/islsm. 14-OR-17

Asagai, Y. (2016). 24 years' experience of low level laser therapy (LLLT) for children with cerebral palsy. International Journal of Physical Medicine \& Rehabilitation, 4, 320-321. doi: 10.4172/2329-9096.1000320

Asagai, Y., Sujaritpong, T., Tranvan, L., \& Ohshiro, T. (2007). Assessment of changes in carotid blood flow following LLLT of the neck. Laser Therapy, 16, 127-132. doi: 10.5978/islsm.16.127

Asagai, Y., Yamamoto, K., Ohshiro, T., \& Ohshiro, T. (2012). Bone metabolism in cerebral palsy and the effect of LightEmitting Diode (LED) irradiation. Laser Therapy, 21, 23-31. doi: 10.5978/islsm.12-OR-051

Asagai, Y., Yamamoto, K., Ohshiro, T., \& Ohshiro, T. (2014). Investigation regarding prevention of insufficiency fractures in children with severe cerebral palsy by Light-Emitting Diode (LED) irradiation. Laser Therapy, 23, 121-128. doi: 10.5978/islsm.14-OR-10

Barbaeva, S.N., Kulishova, T.V., Eliseev, V.V., \& Radchenko, N.V. (2014). The comparative effectiveness of the application of various methods for electrical muscle stimulation in the patients presenting with juvenile cerebral palsy. Voprosy Kurortologii, Fizioterapii i Lechebnoi Fizicheskoi Kultury, 4, 43-46.

Chung, J.H., Kang, D.H., Jo, J.K., \& Lee, S.W. (2012). Assessing the quality of randomized controlled trials published in the Journal of Korean Medical Science from 1986 to 2011. Journal of Korean Medical Science, 27, 973-980. doi: 10.3346/jkms.2012.27.9.973

Chung, W., Lee, K.W., Hwang, I.H., Lee, D.H., \& Kim, S.Y. (2009). Quality assessment of randomized controlled trials in the Journal of the Korean Academy of Family Medicine. Korean Journal of Family Medicine, 30, 626-631. doi: 10.4082/kjfm.2009.30.8.626

Cirović, D., Petronić, I., Nikolić, D., Brdar, R., Pavićević, P., \& Knezević, T. (2009). Effects of electrotherapy in treatment of neurogenic bladder in children with occult spinal dysraphism. Srpski Arhiv za Celokupno Lekarstvo, 137, 502-505. doi:10.2298/SARH0910502C

de Sá, M.R., \& Gomes, R. (2013). Child health promotion: a physiotherapeutic approach. Ciencia \& Saude Coletiva, 18, 1079-1088. doi: 10.1590/S1413-81232013000 400021

Durairaj, S., Dhaneshkumar, K.U., Rajasenthil, K., \& Thakur, A. (2018). A study to find the effectiveness of cryotherapy with task oriented training in improving upper limb function in hemiplegic cerebral palsy. International Journal of Phys- 
iotherapy and Research, 6, 2595-2599. doi: 10.16965/ijpr. 2017.260

El-Maksoud, G.M.A., Sharaf, M.A., \& Rezk-Allah, S.S. (2011). Efficacy of cold therapy on spasticity and hand function in children with cerebral palsy. Journal of Advanced Research, 2, 319-325. doi: 10.1016/j.jare.2011.02.003

Filina, N., Bolotova, N., Manukyan, V., Nikolaeva, O., \& Kompaniets O. (2010). Transcranial magnetotherapy in the treatment of psychoautonomic disorders in children with type 1 diabetes mellitus. Neuroscience and Behavioral Physiology, 40, 1029-1033. doi: 10.1007/s11055-010-9364-7

Galea, M.P. (2012). Physical modalities in the treatment of neurological dysfunction. Clinical Neurology and Neurosurgery, 114, 483-488. doi: 10.1016/j.clineuro.2012.01.009

Garcin, B. (2018). Motor functional neurological disorders: An update. Revue Neurologique, 174, 203-211. doi: 10.1016/j.neurol.2017.11.003

Gawad, H.A.A, Karim, A.E.A, \& Mohammed, A.H. (2015). Shock wave therapy for spastic plantar flexor muscles in hemiplegic cerebral palsy children. Egyptian Journal of Medical Human Genetics, 16, 269-275. doi: 10.1016/j.ejmhg.2014.12.007

Gonkova, M.I., Ilieva, E.M., Ferriero, G., \& Chavdarov, I. (2013). Effect of radial shock wave therapy on muscle spasticity in children with cerebral palsy. International Journal of Rehabilitation Research, 36, 284-290. doi: 10.1097/MRR.0b013e328360e51d

Gottschling, S., Meyer, S., Gribova, I., Distler, L., Berrang, J., Gortner, L., Graf, N., \& Shamdeen M.G. (2008). Laser acupuncture in children with headache: A double-blind, randomized, bicenter, placebo-controlled trial. Pain, 137, 405-412. doi: 10.1016/j.pain.2007.10.004

Gurova, N.Y., \& Babina, L.M. (2007). Efficacy of dynamic magnetotherapy with modulation frequency $10 \mathrm{~Hz}$ in the complex of spa rehabilitation of children with cerebral palsy. Voprosy Kurortologii, Fizioterapii i Lechebnoi Fizicheskoi Kultury, 4, 29-32.

Gurova, N.Y., \& Babina, L.M. (2008). Use of magnetophoresis of glutamic acid and magnesium sulfate in the rehabilitation of children with cerebral palsy. Neuroscience and Behavioral Physiology, 38, 947-951.

Hawrylak, M. (2012). MoŻliwości wykorzystania hydromasaŻu podwodnego w leczeniu spastyczności u dzieci z mózgowym poraŻeniem dziecięcym. [Possibilities of using underwater hydromassage in the treatment of spasticity in children with cerebral palsy] (in Polish). Rehabilitacja Praktyczna, 2, 51-57.

Hawrylak, M. (2012). MoŻliwości wykorzystania hydromasaŻu podwodnego w leczeniu spastyczności u dzieci z mózgowym poraŻeniem dziecięcym- cz.II. [Possibilities of using underwater hydromassage in the treatment of spasticity in children with cerebral palsy- part II] (in Polish). Rehabilitacja Praktyczna, 3, 53-56.

Jadad, A.R., Moore, R.A., Carroll, D., Jenkinson, C., Reynolds, D.J., Gavaghan, D.J., \& McQuay, H.J. (1996). Assessing the quality of reports of randomized clinical trials: is blinding necessary? Controlled Clinical Trials, 17, 1-12. doi: 10.1016/0197-2456(95)00134-4

Jadad scale for reporting randomized controlled trials. Appendix. Evidence-based Obstetric Anesthesia. Edited by Stephen H. Halpern, M. Joanne Douglas Copyright (C) 2005, Blackwell Publishing Ltd.

Kaczyński, Ł., \& Solnica, B. (2012). Pragmatic randomized controlled trials as a source of data in the assessment of effectiveness of medical technology. Przeglad Lekarski, 69, 703-707.

Kajbafzadeh, A.M., Sharifi-Rad, L., Ladi Seyedian, S.S., \& Masoumi, A. (2014). Functional electrical stimulation for management of urinary incontinence in children with myelomeningocele: a randomized trial. Pediatric Surgery International, 30, 663-668. doi: 10.1007/s00383-014-3503-0

Kajbafzadeh, A.M., Sharifi-Rad, L., Nejat, F., Kajbafzadeh, M., \& Talaei, H.R. (2011). Transcutaneous interferential electrical stimulation for management of neurogenic bowel dysfunction in children with myelomeningocele. International Journal of Colorectal Disease, 27, 453-458. doi: 10.1007/s00384-0111328-z

Kwiecień-Czerwieniec, I., \& Woldańska-Okońska, M. (2012). Magnetotherapy in comprehensive pediatric rehabilitation. Polish Annals of Medicine, 19, 163-169. doi: 10.1016/j.poamed.2012.06.006

Lee, J.Y., Chung, J.H., Kang, D.H., Lee, J.W., Moon, H.S., Yoo, T.K., Choi, H.Y., \& Lee, S.W. (2011). Quality assessment of randomized controlled trials published in the Korean Journal of Urology over the past 20 years. Korean Journal of Urology, 52, 642-646. doi: 10.4111/kju.2011.52.9.642

Mancini, M.C., Cardoso, J.R., Sampaio, R.F., Costa, L.C., Cabral, C.M., \& Costa, L.O. (2014). Tutorial for writing systematic reviews for the Brazilian Journal of Physical Therapy (BJPT). Brazilian Journal of Physical Therapy, 18, 471-480. doi: 10.1590/bjpt-rbf.2014.0077

McGough, J.J., Loo, S.K., Sturm, A., Cowen, J., Leuchter, A.F., \& Cook, I.A. (2015). An eight-week, open-trial, pilot feasibility study of trigeminal nerve stimulation in youth with attentiondeficit/hyperactivity disorder. Brain Stimulation, 8, 299-304. doi: 10.1016/j.brs.2014.11.013

Möller, H.J. (2011). Effectiveness studies: advantages and disadvantages. Dialogues in Clinical Neuroscience, 13, 199207.

Nikolaeva, N.V., Bolotova, N.V., Luk'yanov, V.F., Raigorodskii, Y.M., \& Tkacheva, E.N. (2010). Non-pharmacological correction of impaired microcirculation in children with diabetic polyneuropathy. Neuroscience and Behavioral Physiology, 40, 347-350. doi: 10.1007/s11055-010-9262-z

Olchowik, B., Sobaniec, W., Sołowiej E., \& Sobaniec P. (2009). Clinical aspects of spasticity treatment. Neurologia Dziecieca, 18, 47-57.

Olchowik, B., Sobaniec, W., \& Sobaniec, P. (2010). Clinical aspects of application of physical therapy modalities in children. Neurologia Dziecięca, 19, 79-88.

Olivo, S.A., Macedo, L.G., Gadotti, I.C., Fuentes, J., Stanton, T., \& Magee, DJ. (2008). Scales to assess the quality of randomized controlled trials: a systematic review. Physical Therapy, 88 , 156-175. doi: 10.2522/ptj.20070147

Panina, O.S., Zaitseva, G.A., \& Pokazanieva, S.A. (2012). Rehabilitation of neonates with perinatal injuries to the central nervous system using transcranial magnetotherapy. Neuroscience and Behavioral Physiology, 42, 745-747. doi: 10.1007/s11055012-9628-5

Phillips, D., Case, L.E., Griffin, D., Hamilton, K., Lara, S.L., Leiro, B., Monfreda, J., Westlake, E., \& Kishnani, P.S. (2016). Physical therapy management of infants and children with hypophosphatasia. Molecular Genetics and Metabolism, 119, 14-19. doi: 10.1016/j.ymgme.2016.06.010

Simon, S.D. (2006). Statistical evidence in medical trials: what do the data really tell us?. Oxford: Oxford University Press. 
Shukla K.V, Bai A, Milne, George Wells S. (2007). Systematic Review of Quality Assessment Instruments for Randomized Control Trials: Selection of SIGN50 Methodological Checklist. In: Evidence-based health care for all. Abstracts of the 15th Cochrane Colloquium; 2007 23-27 Oct; Sao Paulo, Brazil.

Solopova, I.A., Sukhotina, I.A., Zhvansky, D.S., Ikoeva, G.A., Vissarionov, S.V., Baindurashvili, A.G., Edgerton, V.R., Gerasimenko Y.P., \& Moshonkina, T.R. (2017). Effects of spinal cord stimulation on motor functions in children with cerebral palsy. Neuroscience Letters, 639, 192-198. doi: 10.1016/j.neulet.2017.01.003

Spodaryk, K., \& Bromboszcz, J. (2004). Physical modalities in therapy- the need for scientific research. Medical Rehabilitation, 8, 8-14.

Vidal, X., Morral, A., Costa, L., \& Tur, M. (2011). Radial extracorporeal shock wave therapy (rESWT) in the treatment of spasticity in cerebral palsy: a randomized, placebocontrolled clinical trial. Neurorehabilitation, 29, 413-419. doi:10.3233/NRE-2011-0720

Woszczak, M., śliwiński, Z., Kiljański, M., Kiebzak, W., Szczepanik, M., \& Tomaszewski, W. (2007). Assessment of the efficacy of local cryotherapy in children with cerebral palsy. Fizjoterapia Polska, 3, 275-285.

Yang, H. (2015). Observation on therapeutic effect of half puncture plus transcutaneous acupoint electric stimulation for infantile facial paralysis. Journal of Acupuncture and Tuina Science, 13, 156-159.

Zwolińska, J. (2011). Physical therapy as part of physiotherapy. Przeglad Medyczny Uniwersytetu Rzeszowskiego i Narodowego Instytutu Leków w Warszawie, 9, 278-286. 